\title{
Comparative assessment of the parameters of acute toxicity of natural products
}

\author{
Fazil A. Medetkhanov*, Kseniya V. Muravyova, Dmitriy P. Khadeev, Irina A. Konakova, and Elmira S. Yarullina \\ Kazan State Academy of Veterinary Medicine named after N.E. Bauman, Kazan 420029, Russia
}

\begin{abstract}
The paper presents the results of comparative assessment of the parameters of acute toxicity of complex plant agents under such laboratory codes as CDP, Bronchelp and a phytopropoletic agent KNA for parenteral application, as well as a complex agent KV for use per os. The studies revealed that the single intramuscular administration of agents from natural components CDP, Bronchelp and KNA to laboratory rats in the maximum permissible dose do not have a general toxic effect. Similar results were obtained by oral use of a complex agent KV. According to the results, the tested agents are classified as low-hazardous substances (class 4).
\end{abstract}

\section{Introduction}

In the last two decades, a huge amount of research has focused on developing a replacement for antibiotics to maintain animal health and productivity $[1,2]$. The list of the most deeply studied substitutes includes probiotics, prebiotics, oxidants, plant extracts and such nutraceuticals as copper and zinc, selenium and other macro- and microelements [3-6].

In order to increase the list of medicines that can act as an alternative to antibiotics used in livestock breeding today, we have developed new drugs based on available natural components under the laboratory codesCDP, KNA, Bronchelp and KV taking into account data obtained from literary sources. These agents are theoretically justified and provide for use in veterinary medicine for productive qualities of animals. However, successful introduction of new medicamentous therapy into clinical practice requires high degree of efficiency and safety in the use of new drugs proved according to modern requirements [7].

Wild plant species are widely spread in medicine and veterinary science. Pharmacologically active substances contained in some of them can be used as therapeutic agents in a number of pathologies of domestic and farm animals. Their use can increase stress resistance of some animals thus preventing depletion of the body's compensation abilities. Besides, according to a number of researchers, preparations based on natural raw materials are more effective and have minimum side effects when used as galenicals and parenterally [8, 9]. In view of the above, we have developed a number of agents using affordable plant raw materials and propolis.

The purpose of the study was to conduct a comparative assessment of acute toxicity parameters of various natural agents intended for use in veterinary practice.

\section{Materials and methods}

In this series of tests, two control and 4 test groups of white rats of both sexes, 10 animals each with a body weight of $230-260 \mathrm{~g}$, were formed to determine the acute toxicity [7]. Females were kept separate from males. Clinically healthy animals of the same age, which were under the same feeding and management conditions, were used in the experiment. The animals were added to the test after being pre-quarantined for 14 days.

In order to determine the acute toxicity, the test substances were administered in a single dose to $5.0 \mathrm{ml}$ test groups, orally and intramuscularly. This dose is the maximum permissible for rats with a given body weight [7].

The rats of the first experimental group were exposed to intramuscular administration of a drug under laboratory code CDP, the second - KNA, the third Bronchelp, for which disposable sterile syringes of $10 \mathrm{ml}$ capacity were used, individually for each rat. The rats of the fourth test group were administered KV peros using a reusable injection syringe, disassembled 5-1-10:100-A$\mathrm{M}$, replacing the needle for injection with an olive needle at the end. The fifth group of animals served as control and received distilled water internally via a probe. The sixth group of animals was also controlled and inoculated with $5.0 \mathrm{ml}$ of $0.9 \%$ sterile saline.

All intramuscular agents were sterilized by triplication. Animals were deprived of food 12 to 15 hours prior to the experiment, but they had free access to water. The next food was given 5 hours after the use of test agents. The animals were monitored over 14 days, and were kept under continuous control for the first 24 hours.

In order to assess the acute toxicity pattern, the general state of the animal body, dynamics of respiratory

Corresponding author: ffazilak2@mail.ru 
and body weight readings, changes in their behavior, neuromuscular excitability, time of intoxication onset, and its character, as well as the death of animals were important. Weighing of test animals was carried out immediately before and after the start of the experiment. At the end of the experiment, the animals were euthanized with diethyl ether for macroscopic examination of internal organs.

The animals were opened along the white line of abdomen and along the rib arc, thus ensuring the maximum access to internal organs. The attention was paid to the location of organs, their size and color, tissue elasticity, blood filling of splanchnic vessels. After preliminary assessment of internal organs, the organs were weight using the general laboratory balance.

\section{Research results}

Studies found that parenteral and intragastric administration of test agents in the maximum permissible volume $(5.0 \mathrm{ml})$ to experimental animals did not cause their death. However, some changes in the behaviorof the rats of the test groups were observed, which was particularly evident for animals of the fourth test group that received the KV agent per os (Figure 1). During the first hours after intragastric administration of the developed drug the rats of this group were hiding in a corner, pressed the peritoneum to the base of a cage, bent their back, after which there was a wave-like contraction of the body from thoracic to abdominal part. Breathing in the first minutes was sharply slowed down, up to apnea, which lasted 5 to 6 seconds, followed by a prolonged breath. These respiratory changes lasted 15-20 minutes. Subsequently, breathing became somewhat uniform, but was reliably below physiologically permissible boundaries. So, in two-three hours after oral administration of $\mathrm{KV}$ the number of respiratory movements was lower than in control analogs by $40.0 \%$ at $\mathrm{p} \leq 0.05$.

In the first, second and third test groups, where animals received CDP, KNA and Bronchelp intramuscularly, the intoxication pattern was less pronounced, but the rats were also characterized by some reduction of respiratory movements in dynamics, however the apnea phenomenon as in animals of the fourth test group was not observed. The rats were also hiding in the corner, went off feed, and were suppressed for some time immediately after inoculation of the drug. Three hours after the start of the test, the number of respiratory movements of the animals in the first three test groups was lower than in the control analogues, but higher than of the rats of the fourth test group.

The clinical picture was the same in rats of both control groups receiving distilled water orally and saline parenterally. Immediately after administration of control liquids in either way the rats in these groups became slow-movingandwere also herding together in the corner. Bradypnoeawas observed in animals of the control group with oral administration of distilled water compared to that of rats in the group where sterile saline was used intramuscularly. However, the difference between the groups was not reliable.

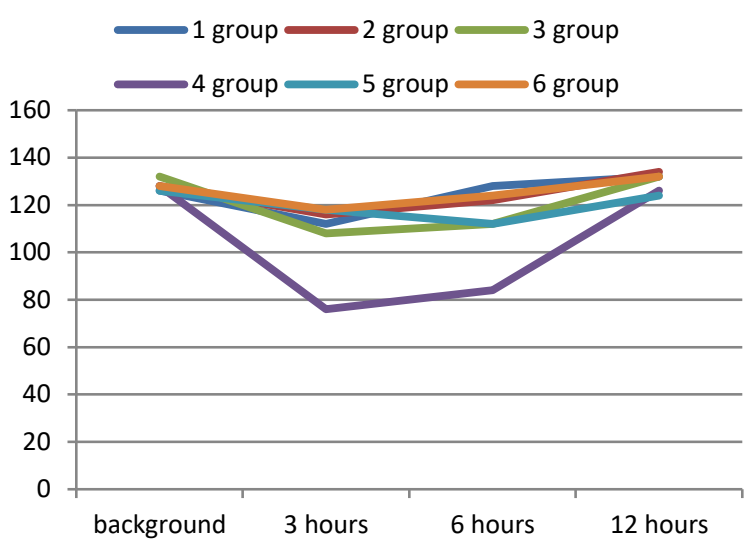

Fig. 1. Respiratory indices of rats using natural agents

On the sixth hour of the experiment, a gradual increase in the number of respiratory movements was noted, but in the fourth pilot group this figure continued to be significantly lower than in the comparison group. When comparing this indicator in the remaining groups with parenteral administration of agents providing for their intramuscular use, there is no difference between the groups.

After 12 hours from the start of the test, the breathing rates between the groups were comparable and there were no differences. Breathing management of animals in test groups up to background values, which were leveled with reference values and lied within the physiological norm, was noted. The rats became mobile, food excitability, act of defecation and urination, and reflex reactions were preserved. The hair of rats remained clean and shiny. The color of the mucous membranes of visible body areas remained unchanged.

During the experiment, changes in animal weight gain were observed, which did not reveal any fundamental differences between the rats of the test and control groups. The use of test substances in the maximum permissible dose at different administration methods did not lead to the death of test animals. Theoretically, the doses used could be tolerated or lethal. Lethal dose of substance $\left(\mathrm{LD}_{50}\right)$ - complex value. Its importance is influenced by resorption, distribution, biotransformation, toxicant extraction, interaction with biologic targets and formation of toxic process.

Each of these factors can significantly influence the behavior of foreign compounds in warm blooded animals. Biological effects caused by chemicals are generally time-limited. One of the main reasons for this is their elimination from the body, which reduces the concentration of substances in blood, organs and tissues. Therefore, in case of toxic origin in test agents, one of the possible manifestations of their negative effect on living structures could be themacroscopic disorder, as well as change in the weight of organs. Considering this fact, we have assessed the state of some internal organs of euthanized rats. 
In case of pathoanatomical autopsy of dead rats of the test and control groups after 14 days of the experiment, in order to study the toxic effect of analyzed agents on the site of administration and internal organs, no visible changes in the macroscopic pattern were established. The arrangement of organs in abdominal and thoracic cavity was correct and they had the natural color. Weighing of some internal organs showed no difference in weight between them depending on the routes of administration of test agents (Table 1).

Table 1 shows that their administration to test rats intramuscularly or orally was not accompanied by an increase or decrease in organ weight, and all parameters between the same organs were consistent with physiological parameters given in special literature [10].

Table1. Weight of internal organs of rats using natural agents

\begin{tabular}{|c|c|c|c|c|c|}
\hline \multirow{2}{*}{ Group } & \multirow{2}{*}{$\begin{array}{c}\text { Body } \\
\text { weight, g }\end{array}$} & \multicolumn{4}{|c|}{ Organ weight, g } \\
\cline { 3 - 6 } & Lungs & Heart & Liver & Kidney \\
\hline 1 & $230-260$ & $2.21 \pm 0.11$ & $1.09 \pm 0.14$ & $13.2 \pm 0.16$ & $1.93 \pm 0.14$ \\
\hline 2 & $230-260$ & $2.27 \pm 0.13$ & $1.17 \pm 0.12$ & $13.1 \pm 0.13$ & $1.90 \pm 0.11$ \\
\hline 3 & $230-260$ & $2.33 \pm 0.12$ & $1.12 \pm 0.14$ & $12.9 \pm 0.22$ & $1.94 \pm 0.12$ \\
\hline 4 & $230-260$ & $2.26 \pm 0.27$ & $1.09 \pm 0.10$ & $13.2 \pm 0.47$ & $1.96 \pm 0.08$ \\
\hline 5 & $230-260$ & $2.33 \pm 0.09$ & $1.06 \pm 0.12$ & $13.3 \pm 0.22$ & $1.96 \pm 0.14$ \\
\hline 6 & $230-260$ & $2.32 \pm 0.17$ & $1.11 \pm 0.15$ & $12.7 \pm 0.24$ & $1.92 \pm 0.12$ \\
\hline
\end{tabular}

Blood is one of the indicators of the body state under the action of exogenous factors, as well as endogenous metabolites produced by organs and tissues in the failure of compensatory mechanisms.

Blood together with lymph forms the internal environment of the body, and by contacting all tissues and cells, it provides their vital activityserving a mediator between the external environment and cells.

Homeostasis and unity of the body are ensured by the nervous system and blood. Therefore, pathogenic factors can cause both qualitative and quantitative changes in blood composition. Considering this fact, we assessed the blood condition taking into accounthematological indices and its morphological composition.

Table 2 shows the results of blood composition study.

Table 2. Hematology of white rats using natural agents

\begin{tabular}{|c|c|c|c|c|}
\hline \multirow{2}{*}{ Group } & \multicolumn{4}{|c|}{ Indicator } \\
\cline { 2 - 5 } & $\begin{array}{c}\text { RBC, } \\
\times 10^{12} / 1\end{array}$ & $\begin{array}{c}\text { WBC, } \\
\times 10^{9} / 1\end{array}$ & $\begin{array}{c}\mathrm{Hb}, \\
\mathrm{g} / \mathrm{l}\end{array}$ & $\begin{array}{c}\text { ESR, } \\
\mathrm{mm} / \mathrm{h}\end{array}$ \\
\hline 1 & $5.42 \pm 1.1$ & $7.16 \pm 0.66$ & $112.4 \pm 2.14$ & $1.1 \pm 0.20$ \\
\hline 2 & $5.63 \pm 1.02$ & $7.22 \pm 1.34$ & $111.0 \pm 2.28$ & $0.98 \pm 0.24$ \\
\hline 3 & $5.44 \pm 0.91$ & $6.95 \pm 1.42$ & $108.2 \pm 2.34$ & $1.2 \pm 0.40$ \\
\hline 4 & $5.44 \pm 1.12$ & $7.08 \pm 1.24$ & $114.2 \pm 2.64$ & $0.86 \pm 0.22$ \\
\hline 5 & $5.28 \pm 0.88$ & $7.12 \pm 0.82$ & $106.6 \pm 2.23$ & $1.12 \pm 0.40$ \\
\hline 6 & $5.32 \pm 0.84$ & $6.98 \pm 1.14$ & $108.2 \pm 2.64$ & $0.96 \pm 0.43$ \\
\hline
\end{tabular}

The above table shows that the number of erythrocytes between groups did not differ significantly. At the same time, their number in the test groups, where various agents derived from plant components were used, was slightly higher than that of the control analogues and was slightly higher than $5.4 \times 10^{12} / 1$ in the first, third and fourth groups. In the second group, where the agent under the laboratory code KNA was used, the red blood cell content was within the range of $5.63 \pm 1.02$ and in the control - from 5.28 to $5.32 \times 10^{12} / 1$.

The number of white blood cells, which mainly perform the protective function from pathogens of various genesis, also had no differences between groups on quantitative structure at $\mathrm{p} \geq 0.05$.

The content of such a chromoprotein as hemoglobin ranged in erythrocytes from 106.4 to $114.2 \mathrm{~g} / \mathrm{l}$, where the lowest amount was observed in blood in rats of the fifth control group and the highest in the fourth experimental group, but the difference between the groups was unreliable. The hematocrit number in all groups was the same and fell within physiological values (Figure 2). The difference between the groups was $1.5-2.0 \%$.

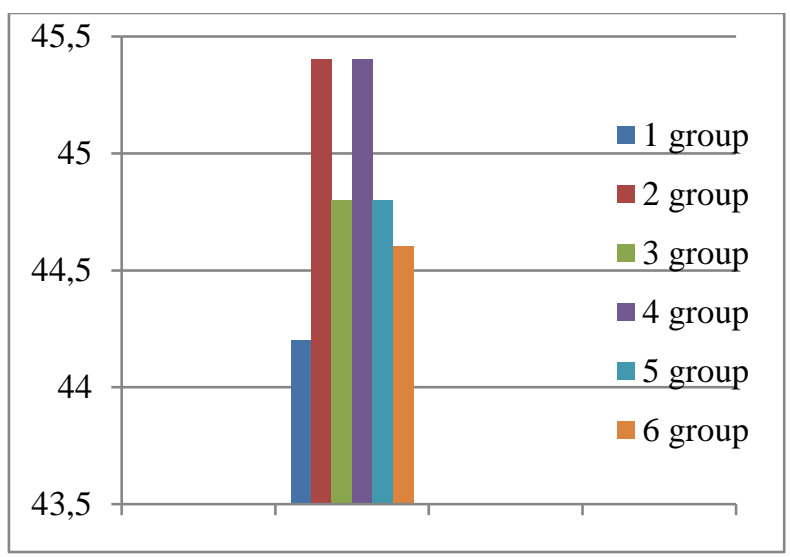

Fig. 2. Hematocritic blood number of white rats using natural agents (\%)

It is well known that the erythrocyte sedimentation rate (ESR) also depends on the change in the ratio of different fractions of blood proteins, on the volume, number and diameter of erythrocytes, on the content of lecithin and blood cholesterol, on the change of relative density of erythrocytes, on bloodviscosity.

The erythrocyte sedimentation rateis greatly influenced by the administration of certain drugs, where the drugs of specific and non-specific irritant therapy, vaccine therapy, hemotransfusioncontribute to its rate, whilesalicyl, mercury and calcium drugs, diuretics, hypnotic and antimalarialagents slow ESR down. Inflammatory processes contribute to the acceleration of ESR.

Therefore, it was not possible to assess the effect of the developed agents by this indicator of differences between groups, which characterizes them as not having the above-mentioned negative properties.

Quantitative and qualitative composition of blood depends on functional and pathological body state. Complex biochemical and physiological changes in the body under various pathological conditions and effects of toxicants change the functional state of the hematopoietic system, and thus the composition of blood. Under the influence of a number of humoral factors, the quantitative and qualitative composition of blood changes in a unique way. 
However, it should be borne in mind that the blood pattern reflects the functional state of only hematopoietic organs, not the body as a whole. At the same time, toxins acting on nerve tissue may not always have a significant effect on the hematopoietic system and vice versa. Obviously, in different diseases, the blood composition may be the same, and conversely, the same disease, depending on the functional state of the hematopoietic organs, may give different blood patterns.

This was the basis for studying the response of hematopoietic organs to natural agents that we used. Blood smears were studied to identify possible deviations in leukocyte structure and to extract the leukocyte formula.

Careful study of various cell members of granulocytic series, destructively altered forms of leukocytes in the form of toxic granulosityin cytoplasm, hypersegmentation, did not reveal the fragmentation of neutrophil nuclei and eosinophils. There are also no qualitative changes in the cell structure of agranulocytes.

In the production of leukocyte blood formula of rats treated with agents of natural origin intramuscularly, the compliance of content of all types of cells with physiological values was noted (Table 3 ). Given that the increase in the number of eosinophils most often characterizes the body state as sensitized to various kinds of allergic substances, the quantitative composition of eosinophils in blood smears was of particular interest. The leukogram analysis showed that the minimum number was in the first test group $(2.2 \%)$ and in the remaining groups the number of granulocytes was higher by $0.2-0.3 \%$, but the difference between the groups was not reliable.

In groups where the natural agents were used orally, the number of eosinophils in the control was $0.3 \%$ higher than in the test analogues, however, these values were also not reliable (Table 4).

The ratio of immature and functionally active neutrophils in all groups was the same, both intramuscularly and intramuscularly.

Single administration of agents in the maximum allowable dose to test rats was not accompanied by quantitative changes of lymphocytes and monocytes in blood smears of rats.

Table 3. Leukocyte for mula of test rats blood with intramuscular use of natural agents

\begin{tabular}{|l|l|c|c|c|}
\hline \multirow{2}{*}{ Indicator } & \multicolumn{4}{|c|}{ Group } \\
\cline { 2 - 5 } & 1 & 2 & 3 & 6 \\
\hline Basophiles, \% & 0 & 0 & 0 & 0 \\
\hline Eosinophils, \% & $2.2 \pm 0.4$ & $2.4 \pm 0.1$ & $2.7 \pm 0.1$ & $2.6 \pm 0.1$ \\
\hline Bandedneutrophils, \% & $3.0 \pm 0.3$ & $2.8 \pm 0.4$ & $2.4 \pm 0.2$ & $3.2 \pm 0.2$ \\
\hline $\begin{array}{l}\text { Segmentedneutrophils, } \\
\%\end{array}$ & $22.8 \pm 1.1$ & $24.2 \pm 1.3$ & $23.6 \pm 3.2$ & $23.4 \pm 1.8$ \\
\hline Lymphocytes, \% & $68.0 \pm 2.1$ & $66.4 \pm 2.6$ & $67.5 \pm 2.4$ & $66.6 \pm 2.4$ \\
\hline Monocytes, \% & $4.0 \pm 0.7$ & $4.2 \pm 0.4$ & $3.8 \pm 0.6$ & $4.2 \pm 0.6$ \\
\hline
\end{tabular}

Table 4. Leukocyte for mula of test rats blood with intragastic use of natural agents

\begin{tabular}{|l|c|c|}
\hline \multirow{2}{*}{ Indicator } & \multicolumn{2}{|c|}{ Group } \\
\cline { 2 - 3 } & 4 & 5 \\
\hline Basophiles, \% & 0 & 0 \\
\hline Eosinophils, \% & $1.9 \pm 0.1$ & $2.2 \pm 0.2$ \\
\hline Bandedneutrophils, \% & $3.2 \pm 0.4$ & $2.8 \pm 0.4$ \\
\hline Segmentedneutrophils, \% & $22.4 \pm 1.2$ & $24.4 \pm 1.2$ \\
\hline Lymphocytes, \% & $68.5 \pm 3.4$ & $66.8 \pm 2.1$ \\
\hline Monocytes, \% & $4.0 \pm 0.3$ & $3.8 \pm 0.6$ \\
\hline
\end{tabular}

\section{Conclusion}

Thus, the studies found that all test agents, when used orally and intramuscularly in test animals, do not result in the death of seeded animals at the maximum permissible dose. The pattern of intoxication is weak and is observed by some changes in behavioral activity and functions of respiratory organs mainly in the first minutes and hours after the administration of the agent to animals. In hematological and morphological composition of blood the quantitative and qualitative shifts are not established. Changes in internal organs that could be characterized as toxic effects of test agents were not identified.

In this regard, based on the classification of L.I. Medved, Y.S. Kagan, E.I. Spynu (1968) currently adopted by WHO, the developed agents can be classified as a group of low-toxic substances, and, according to GOST 12.1.007-76 may refer to the $4^{\text {th }}$ hazard categoryby the degree of toxicity - marginally hazardous substances.

\section{References}

1. S.V. Shabunin, Modern principles and approaches to the creation of therapeutic and preventive agents in veterinary medicine, Veter. doctor, 3, 15-18 (2009)

2. B.V. Usha, O.I. Kalnitskaya, Control of antibiotic residues in raw materials and animal products, Veter. Phys., 3, 21-23 (2009)

3. A. Vagichev, Role of belotine in the balance of feed protein, Compound feeds, 4, 32-33 (2003)

4. V.A. Kokarev, A.N. Fedayev, N.I. Gibalkina, Regulation of chromium in bovine diets, Zootechnia, 4, 17-19 (2000)

5. N.D. Kolesnik, S.A. Semenov, N.I. Ivanchenko, Immunostimulatory properties of purple Echinacea, Zootechnia, 12, 16-17 (2004)

6. P.A. Thacker, Alternatives to antibiotics as growth promoters for use in swine production, Thacker J. of Animal Sci. and Biotechnol., 12 (2013)

7. A.N. Mironov, Guide to pre-clinical drug research, Part 1 (Moscow, 2012)

8. A.V. Sergienko, M.V. Shemonaeva, M.P. Nefedieva, T.A. Lysenko, Prospects of studying pharmacological activity of rosehips oil in 
experimental pathology, Clinics, pharmacol. and therapy, 19(6), 86-87 (2010)

9. F.A. Medethanov, Effect of plant-based drug on the growth rate of laboratory white rats during its evaporation, Sci. notes of Kazan State Acad. of Veter. Med. named after N.E. Bauman, 207, 345-348 (2011)
10. T.V. Abrashova, Y.A. Guschin, M.A. Kovaleva, A.V. Rybakov, A.I. Selezneva, A.P. Sokolova, S.V. Hodko, Physiological, biochemical and biometric indices of experimental animal norms, Directory (LEMA, St. Petersburg, 2013) 